\title{
Effects of Flotage on Immersion Indentation Results of Bone Tissue: An Investigation by Finite Element Analysis
}

\author{
Mingxing Zhou, Zunqiang Fan, Zhichao Ma, Yue Guo, \\ Liguo Yang, Long Qian, and Xingdong Sun \\ School of Mechanical Science and Engineering, Jilin University, Changchun 130025, China \\ Correspondence should be addressed to Zunqiang Fan; fanzunq@jlu.edu.cn
}

Received 27 April 2017; Accepted 20 July 2017; Published 11 September 2017

Academic Editor: David Holec

Copyright (c) 2017 Mingxing Zhou et al. This is an open access article distributed under the Creative Commons Attribution License, which permits unrestricted use, distribution, and reproduction in any medium, provided the original work is properly cited.

\begin{abstract}
In reality, nanoindentation test is an efficient technique for probing the mechanical properties of biological tissue that soaked in the liquid media to keep the bioactivity. However, the effects of flotage imposed on the indenter will lead to inaccuracy when calculating mechanical properties (for instance, elastic modulus and hardness) by using depth-sensing nanoindentation. In this paper, the effects of flotage on the nanoindentation results of cortical bone were investigated by finite element analysis (FEA) simulation. Comparisons of nanoindentation simulation results of bone samples with and without being soaked in the liquid media were carried out. Conclusions show that the difference of load-displacement curves in the case of soaking sample and without soaking sample conditions varies widely based on the change of indentation depth. In other words, the nanoindentation measurements in liquid media will cause significant error in the calculated Young's modules and hardness due to the flotage. By taking into account the effect of flotage, these errors are particularly important to the accurate biomechanics characterization of biological samples.
\end{abstract}

\section{Introduction}

It is now appreciated that nanoindentation technique has been successfully applied to characterize elastic and plastic behaviors of biological tissue such as Young's modulus, hardness, and viscoelasticity, when combined with the in situ observation of electron microscope [1-3]. Recently, Young et al. [4] probed the viscoelasticity of a spider's biological vibration filter by utilizing an atomic force microscopy (AFM) technique, and they investigated the elastic modulus over a temperature range of $15-40^{\circ} \mathrm{C}$ at various loading frequencies. Yoo et al. [5] applied an indentation technique to characterize viscoelastic properties of small ocular and orbital tissue specimens, and they developed quantitative models to predict the wide range response of the tissue's biomechanics based on the experimental measures. To maintain the freshness and prevent dehydration, all of experimental biological tissues should be soaked in the liquid media during the indentation. However, the method to keep the bioactivity of biological sample is seldom mentioned in the above-mentioned experiments. And this will lead to the inaccurate characterization of mechanical properties. Nevertheless, Selby et al. [6] conducted the indentation experiment of the contact lens soaked in phosphate buffered saline during the test. They investigated the influence of hydrogel film thickness on the measured elastic modulus. Li et al. [7] utilized the indentation method to characterize the elastic and viscoelastic mechanical properties of brain tissues, and they immersed the tissue sample in the artificial cerebrospinal fluid to maintain its bioactivity during the whole experimental process. However, when conducting the nanoindentation test in the liquid, the indenter will inevitably bear the flotage, and this will result in the inaccurate collection of reaction force about the indenter. Specifically, the effects of flotage in the experimental microenvironment were not investigated in detail in their work. Regarding the fact that precise evaluation of mechanical properties of biological tissue in immersion indentation is based on fitting unloading stage of load-displacement curves and that these curves are determined by accurate collection of contact force between 
the indenter and sample, the influence of flotage caused by the fluid media should be paid more attention to in biomechanical tests.

This paper focuses on the quantitative influence of flotage on the elastic-plastic indentation mechanical properties of a cortical bone specimen. Three kinds of typical indenters were selected for comparative studies of the load-displacement $(P-h)$ curves in traditional nanoindentation and immersion indentation. When several kinds of indenters penetrate into the liquid media, different magnitudes of flotage will be applied to the corresponding indenters resulting from their geometric diversities. Moreover, percent errors of the specimen's elastic modulus and hardness calculated based on the obtained load-displacement $(P-h)$ curves were listed in very intuitive terms to evaluate the effect of flotage. The research may have certain reference values on indentation of bone, brain, and other biological materials in wet environment.

\section{Theory of Traditional Indentation and Immersion Indentation}

2.1. Traditional Indentation. It is well known that the method of nanoindentation analysis to determine Young's modulus $E$ and hardness $H$ proposed by Oliver and Pharr is applicable for bulk specimens [8]. The formula of Oliver and Pharr is established based on the following assumptions [9]:

(i) The material of specimen is homogenous during the deformation process.

(ii) The material of specimen undergoes elastic-plastic deformation on the loading process and only elastic recovery on the unloading process.

(iii) The creep and viscoelastic deformation of the material should be negligible.

Obviously, the method of calculating elastic modulus and hardness is different from traditional method, which can be written as follows $[8,9]$ :

$$
\begin{aligned}
S & =\frac{d p}{d h} \\
h_{c} & =h_{\max }-\frac{\varepsilon \times p_{\max }}{S} \\
A_{c} & =f\left(h_{c}\right) \\
H & =\frac{P_{\max }}{A_{c}} \\
E_{r} & =\frac{\operatorname{sqrt}(\pi) \times S}{\left(2 \beta \times \operatorname{sqrt}\left(A_{c}\right)\right)} \\
\frac{1}{E_{r}} & =\frac{\left(1-v_{s}^{2}\right)}{E_{s}}+\frac{\left(1-v_{i}^{2}\right)}{E_{i}} .
\end{aligned}
$$

In (1) to (6), $S$ is the contact stiffness by fitting the unloading force-displacement curves $[8,9] . P_{\max }$ is the maximum indentation load; $A_{c}$ is the projected contact area under the peak indentation depth. $h_{c}$ is contact depth that is used to calculate the real contact area and is determined by Sneddon's equation [8]. $H$ is the hardness of specimen, $\beta$ is a correction factor that depends on the geometry of indenter, and $\varepsilon$ is a geometry constant that equals 0.75 for a Berkovich indenter [10].

Obviously, the relation between $E$ and $H$ can be determined by the projected contact area $A_{c}$, contact stiffness $S$, Young's modulus and Poisson's ratio of indenter $\left(E_{i}\right.$ and $\left.v_{i}\right)$, contact depth $h_{c}$, reduced modulus $E_{r}$, and indentation load-displacement $(P-h)$ curve. Regarding the traditional indentation testing on a bone specimen, the unloading stage of load-displacement $(P-h)$ curve directly responds to the elastic-plastic deformation behavior and determines the mechanical parameters of materials [11]. According to (1) and (4), $H$ is directly determined by the ratio of $P_{\max }$ to $A_{c}$, and $S$ is defined by the slope of the initial unloading stage of $P$ - $h$ curve (relevant with $h_{\max }$ and residual depth $h_{f}$ ) [8]. Furthermore, on the basis of (2) and (3), $h_{c}$ is determined by maximum indentation depth $h_{\max }$ and $S$ [12], and $A_{c}$ is proportional to the square of $h_{c}$ [9]. According to (5) and (6), the elastic modulus and hardness of the specimen will be easily obtained on the condition that the contact stiffness $S$ and the projected contact area $A_{c}$ are known.

2.2. Immersion Indentation. A general sketch map of immersion indentation experiment is shown in Figure 1(a). Cortical bone specimen is rigidly fixed on the glass slide that soaked in the liquid media. Assuming that the bone specimen itself will not be influenced by the flotage, only the indenter will bear flotage during the whole experiment process. When conducting the immersion indentation, the indenter moves downward bit by bit, and then the indenter will bear flotage exactly at the time when it touches the liquid level. And this flotage will increase along with the increases of immersion depth $h$ during the whole immersion state. Therefore, the maximum load $P_{\max }$ in immersion indentation might be higher than that in traditional indentation due to the inaccurate collection of reaction force on the indenter. Accordingly, the loading and unloading stages of $P$ - $h$ curve in immersion indentation will both exhibit different extent of deviation from the traditional indentation $P-h$ curve. Thus, it will be of great significance to be certain of the effect of flotage on these two kinds $P$ - $h$ curves by a numerical method. Figure 1(b) shows a two-dimensional axisymmetric immersion indentation model, whose Berkovich indenter tip is exactly in touch with the specimen. And $h$ is the measured immersion depth, $h_{\text {flo }}$ is the submergence depth of specimen, and $h_{\text {ind }}$ is the tip height of indenter. The clamping end of the indenter is equivalent to a cylinder and its radius is $R_{a}$. The relationship between the submergence depth of specimen $h_{\text {flo }}$ and the tip height of indenter $h_{\text {ind }}$ will be clearly clarified here.

Obviously, the measured immersion depth $h$ can be considered as the sum of the maximum indentation depth $h_{\max }$ and the submergence depth $h_{\mathrm{flo}}$ : namely, $h=h_{\mathrm{max}}+h_{\mathrm{flo}}$. Moreover, the flotage applied to the indenter will increase along with the increase of immersion depth $h$; thus, the measured immersion depth $h$ can be classified into two different conditions; namely, $h_{\text {flo }} \leq h_{\text {ind }}$ and $h_{\text {flo }}>h_{\text {ind }}$. 


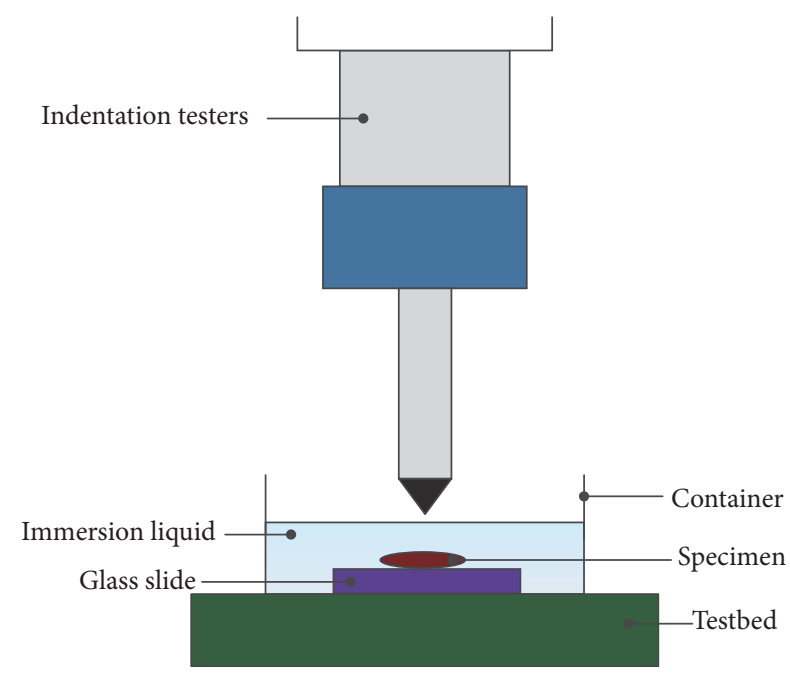

(a)

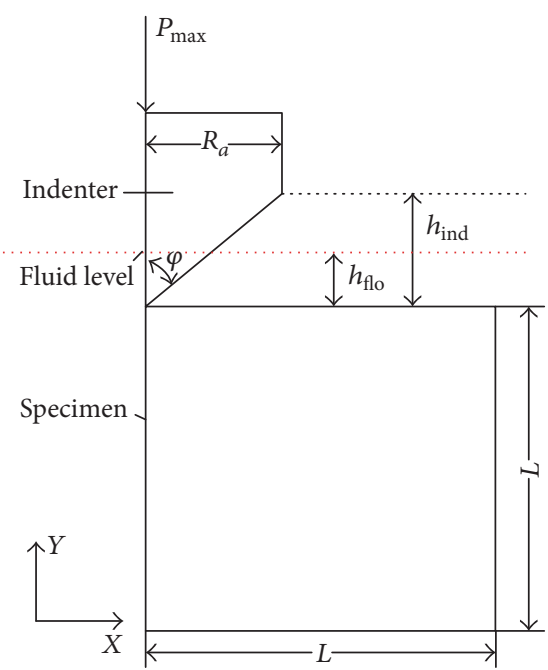

(b)

FIGURE 1: (a) Sketch map of immersion indentation experiment and (b) two-dimensional axisymmetric experiment model of nanoindentation.

TABLE 1: Geometric parameters of different indenters.

\begin{tabular}{lccc}
\hline & Berkovich indenter & Cubic indenter & Spherical indenter \\
\hline $\mathbf{A}_{\mathbf{c}}$ & $24.56 h_{c}{ }^{2}$ & $2.5981 h_{c}{ }^{2}$ & $\pi\left(2 R h_{c}-h_{c}{ }^{2}\right)$ \\
$\boldsymbol{\varphi}$ & $70.32^{\circ}$ & $42.28^{\circ}$ & \\
$\boldsymbol{\alpha}$ & $65.3^{\circ}$ & $35.2644^{\circ}$ & \\
\hline
\end{tabular}

Assuming that the fluid keeps relatively static in the whole indentation process and the liquid media's surface tension adhered to the indenter can be neglected, thus the indenter will only bear the flotage in liquid media. It is clear that the magnitude of this flotage depends on the geometry parameters of indenters, and Table 1 shows some geometry parameters about three indenters used in this paper $[13,14]$. Here, $\varphi$ is the equivalent semicone angle, $\alpha$ is the included angel between indenter's geometry center line and faced plane, and it will be utilized to calculate the indenters' geometric volume that determines the flotage.

Regarding the Berkovich indenter, when the submergence depth $h_{\text {flo }}$ is smaller than the tip height of indenter $h_{\text {ind }}$, namely, $h_{\text {flo }} \leq h_{\text {ind }}$, the formula of flotage can be written as follows:

$$
\begin{aligned}
& P_{\text {flo }}=\sqrt{3} \rho g h^{3} \tan ^{2} \alpha \quad\left(h \leq h_{\text {ind }}\right) \\
& P_{\text {flo }}=\sqrt{3} \rho g h_{\text {ind }}^{3} \tan ^{2} \alpha+\rho g \pi R_{a}^{2}\left(h-h_{\text {ind }}\right)
\end{aligned}
$$

$$
\left(h>h_{\text {ind }}\right) \text {. }
$$

Here, $P_{\text {flo }}$ is the flotage applied to the indenter, $\rho$ is the density of liquid media, $g$ is the ratio of gravity and mass, and $h$ is the measured immersion depth. On the other hand, the formula of flotage can be written as follows when the condition is $h_{\text {flo }}>h_{\text {ind }}$ :

$$
P_{\text {flo }}=\sqrt{3} \rho g h_{\text {ind }}^{3} \tan ^{2} \alpha+\rho g \pi R_{a}^{2}\left(h-h_{\text {ind }}\right) .
$$

Similarly, the flotage applied to other spherical indenter and cubic indenter can be deduced by using trigonometric functions and Archimedes flotage principle. Regarding the spherical indenter, when the submergence depth $h_{\text {flo }}$ is smaller than the tip height of indenter $h_{\text {ind }}$, namely, $h_{\text {flo }} \leq$ $h_{\text {ind }}$, the formula of flotage can be written as

$$
\begin{aligned}
& P_{\text {flo }}=\rho g \pi h^{2}\left(R_{a}-\frac{h}{3}\right) \quad\left(h \leq h_{\text {ind }}\right) . \\
& P_{\text {flo }}=\frac{2}{3} \rho g \pi R_{a}^{3}+\rho g \pi R_{a}^{2}\left(h-h_{\text {ind }}\right) \quad\left(h>h_{\text {ind }}\right)
\end{aligned}
$$

On the other hand, when the condition is $h_{\text {flo }}>h_{\text {ind }}$, the formula of flotage can be written as

$$
P_{\text {flo }}=\sqrt{3} \rho g h_{\text {ind }}^{3} \tan ^{2} \alpha+\rho g \pi R_{a}^{2}\left(h-h_{\text {ind }}\right) .
$$

Regarding the cubic indenter, when the submergence depth $h_{\text {flo }}$ is smaller than the tip height of indenter $h_{\text {ind }}$, namely, $h_{\text {flo }} \leq h_{\text {ind }}$, the formula of flotage can be written as

$$
\begin{aligned}
& P_{\text {flo }}=\sqrt{3} \rho g h^{3} \tan ^{2} \alpha \quad\left(h \leq h_{\text {ind }}\right) \\
& P_{\text {flo }}=\sqrt{3} \rho g h_{\text {ind }}^{3} \tan ^{2} \alpha+\rho g \pi R_{a}^{2}\left(h-h_{\text {ind }}\right) \\
& \quad\left(h>h_{\text {ind }}\right) .
\end{aligned}
$$

On the other hand, when the condition is $h_{\text {flo }}>h_{\text {ind }}$, the formula of flotage can be written as

$$
P_{\text {flo }}=\sqrt{3} \rho g h_{\text {ind }}^{3} \tan ^{2} \alpha+\rho g \pi R_{a}^{2}\left(h-h_{\text {ind }}\right) .
$$

\section{FEA Simulation Results and Discussion}

3.1. Simulation Model. In order to investigate the effects of flotage on the results of immersion indentation, finite element analysis (FEA) was carried out by using the commercial 


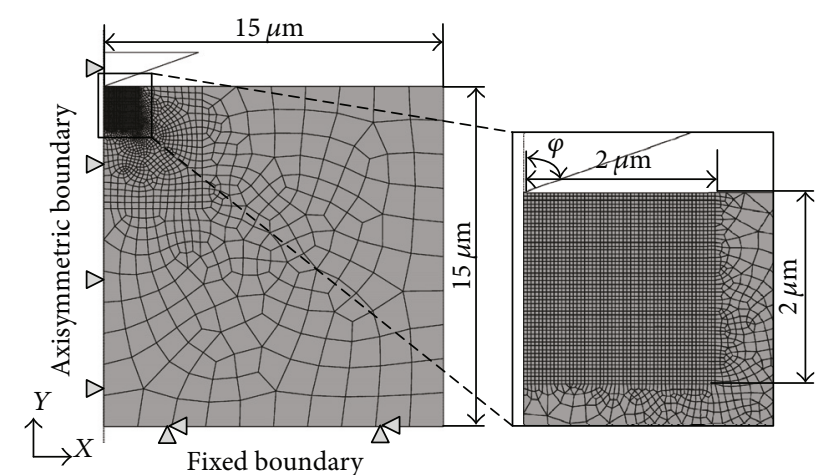

FIGURE 2: The two-dimensional meshed axisymmetric simulation model of nanoindentation.

TABLE 2: Elastic and plastic properties of cortical bone.

\begin{tabular}{lccc}
\hline Material & $\begin{array}{c}\text { Young's } \\
\text { modulus, } \\
E(\mathrm{GPa})\end{array}$ & $\begin{array}{c}\text { Poisson's, } \\
\text { ratio, } \\
v\end{array}$ & $\begin{array}{c}\text { Yield } \\
\text { strength } \\
\sigma_{s}(\mathrm{GPa})\end{array}$ \\
\hline Cortical bone & 13.56 & 0.3 & 0.301 \\
\hline
\end{tabular}

software ABAQUS 6.11 version [15]. Figure 2 showed a twodimensional simulation model of nanoindentation, whose bottom of the specimen was frozen and its left side was applied with axisymmetric boundary conditions. It can be confirmed from previous FEA results that the tip of the indenter can be equivalent to a half cone angle, and the equivalent semicone angle of Berkovich indenter tip was $70.32^{\circ}[13,14]$. The specimen was meshed with four node bilinear axisymmetric reduced integration elements (CAX4R element type), and the whole mesh size was $0.3 \mu \mathrm{m}$. No friction was considered between the indenter and specimen. Due to the large deformations involved in the simulation, adaptive meshing was used. The indenter had only one degree of freedom and was applied with displacement boundary to accomplish the loading and unloading steps. Compared with the sample, the hardness of diamond indenter was extremely large, so the indenter was treated as a rigid body in the simulation. The total size of $2 \mathrm{D}$ axisymmetric meshed simulation model was $15 \times 15 \mu \mathrm{m}$ and the indenter height was set to be $1.5 \mu \mathrm{m}$ here. A homogenous isotropic cortical bone was chosen as the simulation sample and it was treated as ideal elastic-plastic body. The detailed mechanical properties were given in Table 2 [16].

The FEA indentation process of immersion indentation and traditional indentation was divided into two steps of loading and unloading. Both loading and unloading steps were all controlled by the displacement method with the maximum indentation depth of indenter $h_{\max }$ being 200, 400, 600,800 , and $1000 \mathrm{~nm}$. Regarding the immersion indentation, indenters will bear some flotage increasing with the immersion depth $h$, and therefore the indenter will suffer an equivalent flotage load when it immerses into the liquid medium in the simulation. Assuming that the homogeneous liquid media are 3.5\% normal saline and the density of this normal saline is $1.03 \mathrm{~g} / \mathrm{cm}^{3}$, when the submergence depth of specimen $h_{\text {flo }}$ is $2.28 \mathrm{~mm}$, it can be calculated from (7) that the flotage of Berkovich indenter $P_{\text {flo }}$ is $1 \mathrm{mN}$. Similarly, regarding the cubic indenter whose geometry standard is identical with Berkovich indenter, the flotage of it is $0.106 \mathrm{mN}$. According to (9), assuming that the radius of spherical indenter $R_{a}$ is $3 \mathrm{~mm}$, the flotage of this indenter will be $0.377 \mathrm{mN}$.

3.2. Simulation Results. Figure 3 illustrates corresponding $P$ - $h$ curves of three kinds of indenters in the immersion indentation and traditional indentation. It can be clearly seen that the loads at the maximum indentation depth $h_{\max }$ in immersion indentation are higher than that in traditional indentation, and the envelope area of $P$ - $h$ curves in immersion indentation is apparently larger than that in traditional indentation. In addition, the loading and unloading stages of $P$ - $h$ curves both exhibit different extent of deviations from traditional indentation $P-h$ curves. Specifically, when the maximum indentation depth $h_{\max }$ equals $200 \mathrm{~nm}$, the deviations of $P$ - $h$ curves between immersion indentation and traditional indentation become most obvious no matter what kind of indenter is employed in the simulation. That is to say, these deviations will cause inaccuracy of sample's elastic modulus and hardness to a certain extent.

To have a quantitative explanation for the effects of flotage on the results of immersion indentation, the percent errors of elastic modulus and hardness are calculated based on the following equation:

$$
\left\{\begin{array}{l}
\text { Error }_{E} \%=\frac{\left(E_{\mathrm{flo}}-E_{s}\right)}{E_{s}} \times 100 \% \\
\text { Error }_{H} \%=\frac{\left(H_{\mathrm{flo}}-H_{s}\right)}{H_{s}} \times 100 \%
\end{array}\right\}
$$

Here, $E_{\mathrm{flo}}$ is the calculated elastic modulus of immersion indentation and $E_{s}$ is the calculated elastic modulus of traditional indentation. Similarly, $H_{\text {flo }}$ is the calculated hardness of immersion indentation and $H_{s}$ is the calculated hardness of traditional indentation.

As drawn in Figure 4, the percent errors of elastic modulus and hardness for three kinds of indenters in immersion indentation are compared with that of traditional indentation. It is obvious that the percent errors of elastic modulus Error $_{E}$ and percent errors of hardness Error ${ }_{H}$ will decrease with the increase of the maximum indentation depth $h_{\max }$. Specifically, when the maximum indentation depth $h_{\max }$ equals $200 \mathrm{~nm}$, the percent errors of elastic modulus Error ${ }_{E}$ exceed $100 \%$, regardless of the kinds of indenters. Meanwhile, the percent errors of hardness Error $_{H}$ are about $120.6 \%$, $82.7 \%$, and $56.7 \%$, which are obtained from Berkovich indenter, cubic indenter, and spherical indenter, respectively.

Similarly, when the maximum indentation depth $h_{\max }$ increases to $1000 \mathrm{~nm}$, the Error $_{E}$ values decrease to $17.5 \%$, $4.4 \%$, and $10.9 \%$, while the Error $_{H}$ values decrease to $5.8 \%$, $5.1 \%$, and $7.2 \%$ when using Berkovich, cubic, and spherical indenters, respectively. Finally, the percent errors of elastic modulus for cubic indenter have always been the smallest one when compared with other indenters at the same indentation depth $h_{\max }$. 



Tra: traditional indentation Imm: immersion indentation

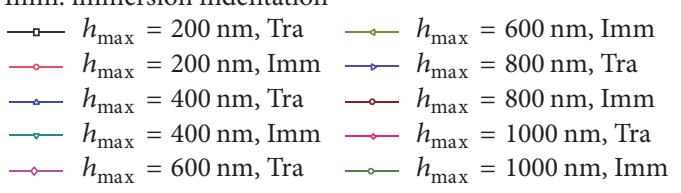

(a)
Tra: traditional indentation Imm: immersion indentation

$$
\begin{aligned}
& \multimap h_{\max }=200 \mathrm{~nm} \text {, Tra } \multimap h_{\max }=600 \mathrm{~nm}, \mathrm{Imm} \\
& \multimap h_{\max }=200 \mathrm{~nm}, \text { Imm } \longrightarrow h_{\max }=800 \mathrm{~nm} \text {, Tra } \\
& \because h_{\max }=400 \mathrm{~nm} \text {, Tra } \multimap h_{\max }=800 \mathrm{~nm}, \mathrm{Imm} \\
& \multimap h_{\max }=400 \mathrm{~nm}, \mathrm{Imm} \multimap h_{\max }=1000 \mathrm{~nm} \text {, Tra } \\
& \multimap h_{\max }=600 \mathrm{~nm} \text {, Tra } \multimap h_{\max }=1000 \mathrm{~nm}, \mathrm{Imm}
\end{aligned}
$$

(b)

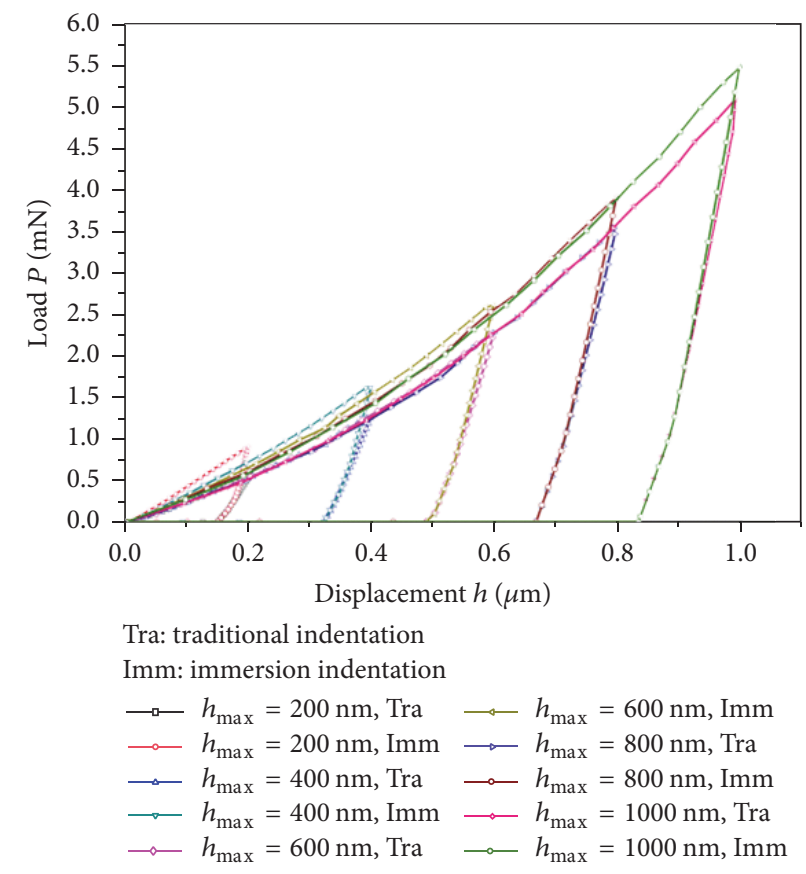

(c)

Figure 3: Load-displacement curves of immersion indentation and traditional indentation with different indenters, (a) cubic indenter, (b) Berkovich indenter, and (c) spherical indenter.

\section{Conclusions}

Regarding the immersion indentation whose specimen is soaked in the liquid media, the effects of flotage on the indentation results used to be neglected, and this will cause the properties such as elastic modulus and hardness of the sample to be somehow inaccurate. In this paper, the effects of flotage on the results of cortical bone nanoindentation were investigated by finite element analysis simulation. Conclusions are drawn as follows:

(1) The comparison studies in $P$ - $h$ curves of immersion indentation and traditional indentation indicate that the flotage could to some extent affect the envelope area of the $P$ - $h$ curves. When under the same indentation depth, the envelope area of immersion 


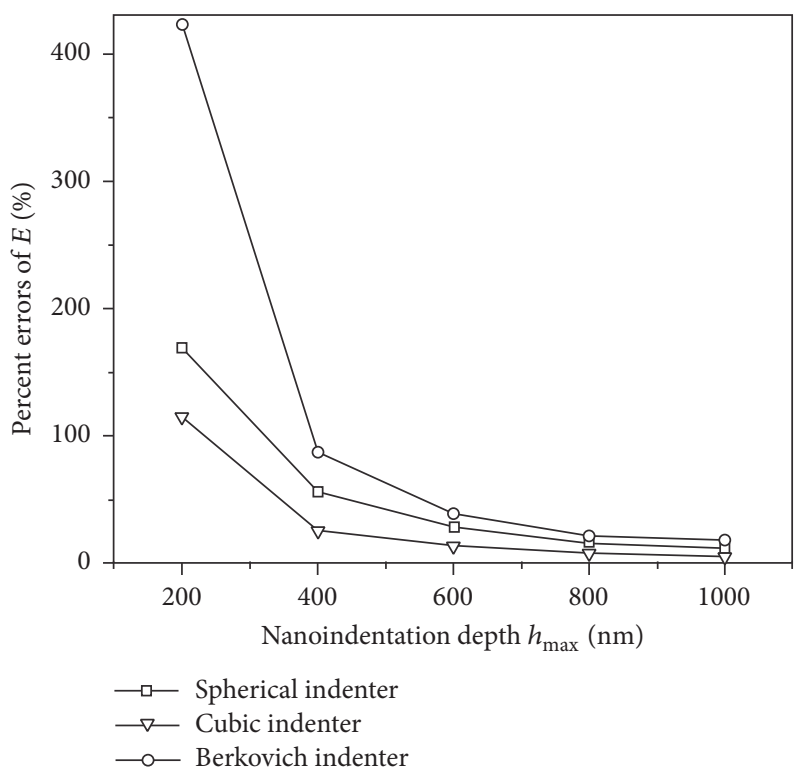

(a)

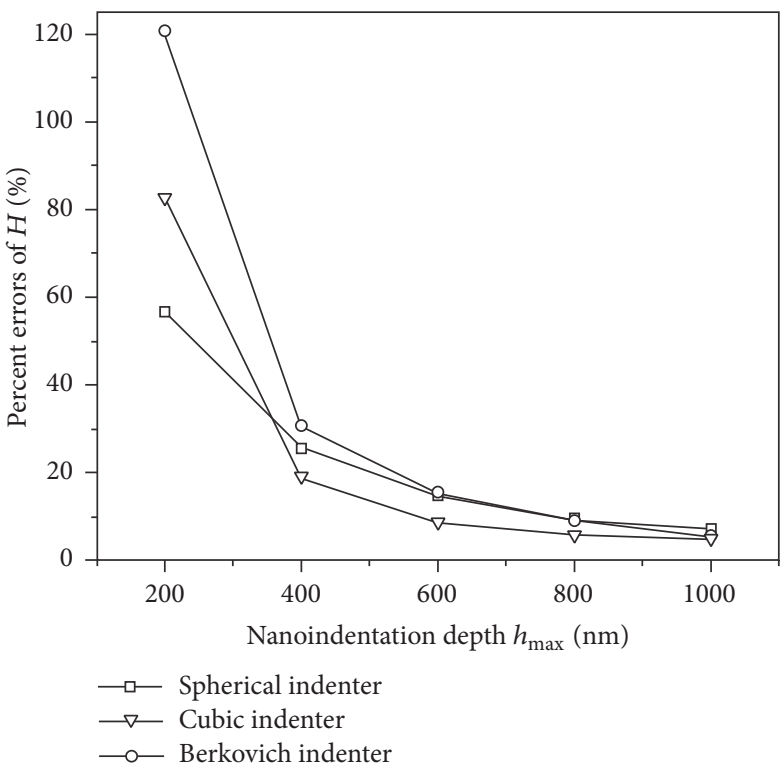

(b)

Figure 4: (a) Percent errors of elastic modulus for different indenters with immersion indentation compared with traditional indentation. (b) Percent errors of hardness for different indenters with immersion indentation compared with traditional indentation.

indentation will be larger than that of traditional indentation.

(2) From the calculated percent errors of elastic modulus and hardness, it is known that when the maximum indentation depth ranges from $200 \mathrm{~nm}$ to $1000 \mathrm{~nm}$, the percent errors of elastic modulus for cubic indenter is the smallest and the Berkovich indenter is the largest one all the time.

(3) With the decrease of maximum indentation depth, the percent errors values of elastic modulus and hardness become larger on the contrary.

\section{Conflicts of Interest}

The authors declare no potential conflicts of interest with respect to the research, authorship, and/or publication of this article.

\section{Acknowledgments}

The authors disclosed receipt of the following financial support for the research, authorship, and/or publication of this article: the National Natural Science Foundation of China (51422503 and 51505180), Fund Guiding on Strategic Adjustment of Jilin Provincial Economic Structure Project (2014Z045), Major Project of Jilin Province Science and Technology Development Plan (20150203014GX, 20150520108JH, and 20170101134JC), Jilin Provincial Industrial Innovation Special Fund Project (2016C030), and Fundamental Research Funds for Central Universities.

\section{References}

[1] A. Opdahl, S. H. Kim, T. S. Koffas, C. Marmo, and G. A. Somorjai, "Surface mechanical properties of pHEMA contact lenses: viscoelastic and adhesive property changes on exposure to controlled humidity," Journal of Biomedical Materials Research - Part A, vol. 67, no. 1, pp. 350-356, 2003.

[2] A. J. Bushby, V. L. Ferguson, and A. Boyde, "Nanoindentation of bone: comparison of specimens tested in liquid and embedded in polymethylmethacrylate," Journal of Materials Research, vol. 19, no. 1, pp. 249-259, 2004.

[3] C. Klapperich, K. Komvopoulos, and L. Pruitt, "Nanomechanical properties of polymers determined from nanoindentation experiments," Journal of Tribology, vol. 123, no. 3, pp. 624-631, 2001.

[4] S. L. Young, M. Chyasnavichyus, M. Erko et al., "A spider's biological vibration filter: micromechanical characteristics of a biomaterial surface," Acta Biomaterialia, vol.10, no. 11, pp. 48324842, 2014.

[5] L. Yoo, J. Reed, A. Shin et al., "Characterization of ocular tissues using microindentation and Hertzian viscoelastic models," Investigative Ophthalmology and Visual Science, vol. 52, no. 6, pp. 3475-3482, 2011.

[6] A. Selby, C. Maldonado-Codina, and B. Derby, "Influence of specimen thickness on the nanoindentation of hydrogels: measuring the mechanical properties of soft contact lenses," Journal of the Mechanical Behavior of Biomedical Materials, vol. 35, pp. 144-156, 2014.

[7] Y. Li, J. Deng, J. Zhou, and X. Li, "Elastic and viscoelastic mechanical properties of brain tissues on the implanting trajectory of sub-thalamic nucleus stimulation," Journal of Materials Science: Materials in Medicine, vol. 27, no. 11, article no. 163, 2016. 
[8] W. C. Oliver and G. M. Pharr, "Improved technique for determining hardness and elastic modulus using load and displacement sensing indentation experiments," Journal of Materials Research, vol. 7, no. 6, pp. 1564-1580, 1992.

[9] W. C. Oliver and G. M. Pharr, "Measurement of hardness and elastic modulus by instrumented indentation: advances in understanding and refinements to methodology," Journal of Materials Research, vol. 19, no. 1, pp. 3-20, 2004.

[10] A. C. Fischer-Cripps, "Nanoindentation Testing," in Nanoindentation, Mechanical Engineering Series, pp. 21-37, Springer New York, New York, NY, 2011.

[11] G. M. Pharr and A. Bolshakov, "Understanding nanoindentation unloading curves," Journal of Materials Research, vol. 17, no. 10, pp. 2660-2671, 2002.

[12] I. N. Sneddon, "The relation between load and penetration in the axisymmetric Boussinesq problem for a punch of arbitrary profile," International Journal of Engineering Science, vol. 3, pp. 47-57, 1965.

[13] L. Min, C. Wei-Min, L. Nai-Gang, and W. Ling-Dong, "A numerical study of indentation using indenters of different geometry," Journal of Materials Research, vol. 19, no. 1, pp. 7378, 2004.

[14] J. L. Hay and G. M. Pharr, "Instrumented Indentation Testing, ASM Handbook, Volume 8," in Proceedings of the Instrumented Indentation Testing, ASM Handbook, Volume 8, 2000.

[15] ABAQUS version 6.9, HIbbitt, Karlsson \&amp; Scorensen, Inc., Pawtucket, RI, 2011.

[16] L. P. Mullins, M. S. Bruzzi, and P. E. McHugh, "Calibration of a constitutive model for the post-yield behaviour of cortical bone," Journal of the Mechanical Behavior of Biomedical Materials, vol. 2, no. 5, pp. 460-470, 2009. 

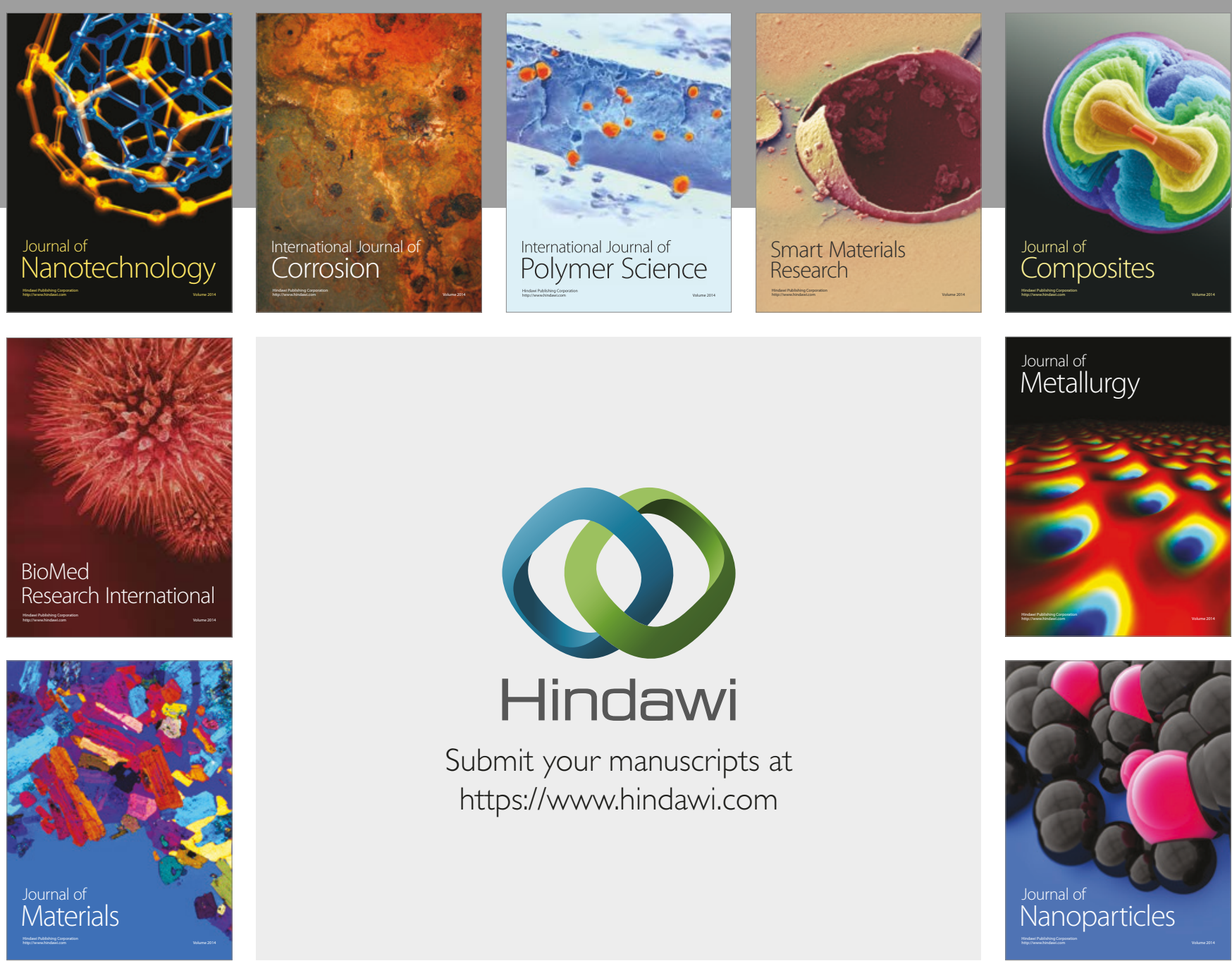

\section{Hindawi}

Submit your manuscripts at

https://www.hindawi.com
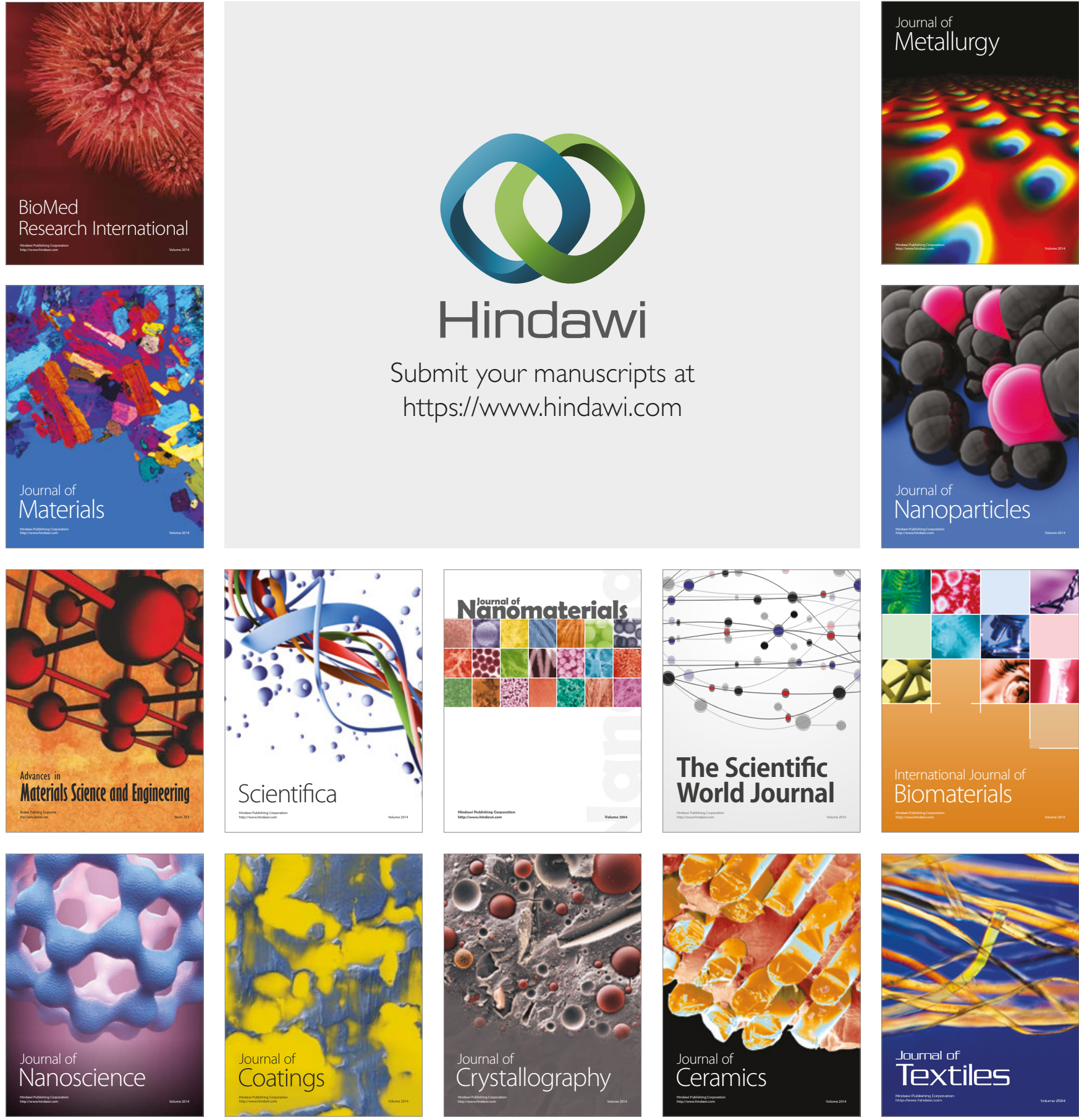

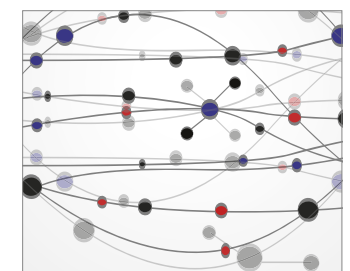

The Scientific World Journal
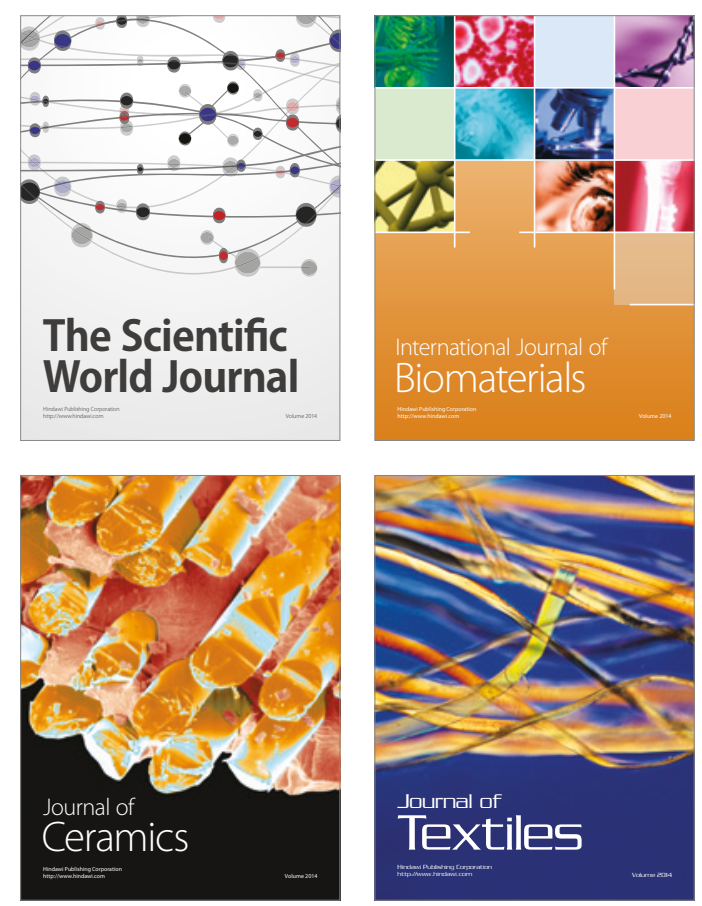